\title{
A NOTE ON SHEAVES WITHOUT SELF-EXTENSIONS ON THE PROJECTIVE $n$-SPACE
}

\author{
DIETER HAPPEL AND DAN ZACHARIA
}

(Communicated by Harm Derksen)

\begin{abstract}
Let $\mathbf{P}^{n}$ be the projective $n$-space over the complex numbers. In this note we show that an indecomposable rigid coherent sheaf on $\mathbf{P}^{n}$ has a trivial endomorphism algebra. This generalizes a result of Drézet for $n=2$.
\end{abstract}

Let $\mathbf{P}^{n}$ be the projective $n$-space over the complex numbers. Recall that an exceptional sheaf is a coherent sheaf $E$ such that $\operatorname{Ext}^{i}(E, E)=0$ for all $i>0$ and End $E \cong \mathbb{C}$. Drézet proved in 4 that if $E$ is an indecomposable sheaf over $\mathbf{P}^{2}$ such that $\operatorname{Ext}^{1}(E, E)=0$ then its endomorphism ring is trivial, and also that $\operatorname{Ext}^{2}(E, E)=0$. Moreover, the sheaf is locally free. Partly motivated by this result, we prove in this short note that if $E$ is an indecomposable coherent sheaf over the projective $n$-space such that $\operatorname{Ext}^{1}(E, E)=0$, then we automatically get that End $E \cong \mathbb{C}$. The proof involves reducing the problem to indecomposable linear modules without self-extensions over the polynomial algebra. In the second part of this article, we look at the Auslander-Reiten quiver of the derived category of coherent sheaves over the projective $n$-space. It is known (11]) that if $n>1$, then all the components are of type $\mathbb{Z} A_{\infty}$. Then, using the Bernstein-Gelfand-Gelfand correspondence ([3]) we prove that each connected component contains at most one sheaf. We also show that in this case the sheaf lies on the boundary of the component.

Throughout this article, $S$ will denote the polynomial ring in $n+1$ variables with coefficients in $\mathbb{C}$. Its Koszul dual is the exterior algebra in $n+1$ variables $x_{0}, x_{1}, \ldots, x_{n}$ which we denote by $R$. It is well known that $R$ is a graded, local, finite dimensional algebra over $\mathbb{C}$. Moreover, $R$ is a self-injective algebra; that is, the notions of free and of injective $R$-modules coincide. Denote by $\operatorname{Lin} R$ and $\operatorname{Lin} S$ the categories of linear $R$-modules (linear $S$-modules respectively), that is, of the modules having a linear free resolution. It is well known that both $\operatorname{Lin} R$ and $\operatorname{Lin} S$ are closed under extensions and cokernels of monomorphisms. We have mutual inverse Koszul dualities:

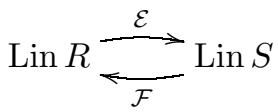

Received by the editors December 4, 2010 and, in revised form, December 20, 2011.

2010 Mathematics Subject Classification. Primary 14F05, 16E10; Secondary 16E65, 16G20, $16 \mathrm{G} 70$.

Key words and phrases. Exterior algebra, projective space, coherent sheaves, vector bundles, exceptional objects.

The second author is supported by the NSA grant H98230-11-1-0152. 
given by $\mathcal{E}(M)=\bigoplus_{n>0} \operatorname{Ext}_{R}^{n}(M, \mathbb{C})$ with the obvious action of $S$ on $\mathcal{E}(M)$ and with the inverse duality $\mathcal{F}$ defined in a similar way.

Let $\bmod ^{Z} R$ be the category of finitely generated graded $R$-modules and graded (degree 0) homomorphisms, where we denote by $\operatorname{Hom}_{R}(M, N)_{0}$ the degree zero homomorphisms from $M$ to $N$. Denote by $\underline{\bmod }^{Z} R$ the stable category of finitely generated graded $R$-modules. The objects of $\underline{\bmod }^{Z} R$ are the finitely generated graded $R$-modules, and the morphisms are given by

$$
\underline{\operatorname{Hom}}_{R}(M, N)_{0}=\operatorname{Hom}_{R}(M, N)_{0} / \mathcal{P}(M, N)_{0}
$$

where $\mathcal{P}(M, N)_{0}$ is the space of graded homomorphisms from $M$ to $N$ factoring through a free $R$-module. The stable graded category $\bmod ^{Z} R$ is a triangulated category where the shift functor is given by the first cosyzygy; that is, every distinguished triangle in the stable category is of the form $A \rightarrow B \rightarrow C \rightarrow \Omega^{-1} A$ where $\Omega: \underline{\bmod }^{Z} R \rightarrow \underline{\bmod }^{Z} R$ denotes the syzygy functor. Note also that the stable category $\underline{\bmod }^{Z} R$ has a Serre duality:

$$
D \underline{\operatorname{Hom}}_{R}\left(X, \Omega^{-m} Y\right)_{0} \cong \underline{\operatorname{Hom}}_{R}\left(Y, \Omega^{m+1} X(n+1)\right)_{0}
$$

for each integer $m$ and all $R$-modules $X$ and $Y$, where $X(n+1)$ is the graded shift of $X$. It is immediate to see that we can derive the well-known Auslander-Reiten formula from the above in the case when $m=1$.

Let $M=\bigoplus M_{i}$ be a finitely generated graded module over the exterior algebra $R$ and let $\xi$ be a homogeneous element of degree 1 in $R$. Following [3], we can view the left multiplication by $\xi$ on $M$ as inducing a complex of $K$-vector spaces

$$
L_{\xi} M: \quad \cdots M_{i-1} \stackrel{\cdot \xi}{\longrightarrow} M_{i} \stackrel{\cdot \xi}{\longrightarrow} M_{i+1} \rightarrow \cdots
$$

We call the graded module $M$ nice or proper if the homology $H_{i}\left(L_{\xi} M\right)=0$ for each $i \neq 0$ and for each homogeneous element $\xi \neq 0$ of degree 1 . It is well known that a graded module $M$ is free if and only if $H_{i}\left(L_{\xi} M\right)=0$ for each $i \in \mathbb{Z}$ and for each nonzero homogeneous element $\xi \in R_{1}$.

The derived category $\mathcal{D}^{b}\left(\operatorname{coh} \mathbf{P}^{n}\right)$ of coherent sheaves over the projective space is also, triangulated, and the BGG correspondence (see [3]

$$
\Phi: \underline{\bmod }^{Z} R \rightarrow \mathcal{D}^{b}\left(\operatorname{coh} \mathbf{P}^{n}\right)
$$

is an exact equivalence of triangulated categories that assigns to each finitely generated graded $R$-module the equivalence class of a bounded complex of vector bundles on $\mathbf{P}^{n}$. If $M=\bigoplus M_{i}$, we use also $M_{i}$ to denote the trivial bundle with finite dimensional fiber $M_{i}$ and we set

$$
\Phi(M): \quad \ldots \longrightarrow \mathcal{O}(i) \otimes M_{i} \stackrel{\delta^{i}}{\longrightarrow} \mathcal{O}(i+1) \otimes M_{i+1} \longrightarrow \ldots
$$

where the differentials $\delta^{i}$ are defined in the following way. Let $x_{0}, x_{1}, \ldots, x_{n}$ be a basis of $V$, and let $\xi_{0}, \xi_{1}, \ldots, \xi_{n}$ denote the dual basis of $V^{*}$. Then $\delta^{i}=\sum_{j=0}^{n} \xi_{j} \otimes x_{j}$. Moreover for each nice $R$-module $M$, the complex $\Phi(M)$ is quasi-isomorphic to the stalk complex of a vector bundle over the projective $n$-space, and all the vector bundles can be obtained in this way from the nice $R$-modules. 
Throughout this article if $M$ is a graded module, then its graded shift, denoted by $M(i)$, is the graded module given by $M(i)_{n}=M_{i+n}$ for all integers $n$. If $M$ is a graded module, we denote its truncation at the $k$-th level by $M_{\geq k}=M_{k} \oplus M_{k+1} \oplus \cdots$.

\section{THE ENDOMORPHISM RING OF A RIGID SHEAF}

Let $F$ be an indecomposable coherent sheaf over the projective $n$-space. Assume also that $F$ is rigid, that is, $\operatorname{Ext}^{1}(F, F)=0$. We prove in this section that the endomorphism ring of $F$ is trivial. This is done by reducing the problem to indecomposable rigid linear modules over the polynomial algebra $S$ in $n+1$ variables.

Let $M$ be a finitely generated, graded, indecomposable nonprojective $S$-module. We say that $M$ has no graded self-extensions if every short exact sequence of graded $S$-modules $0 \rightarrow M \rightarrow X \rightarrow M \rightarrow 0$ splits, that is, $\operatorname{Ext}_{S}^{1}(M, M)_{0}=0$.

In order to prove our next result we recall the following definition and facts from 6]. We say that a module $M \in \bmod ^{Z} S$ has a linear presentation if it has a graded free presentation $P_{1} \rightarrow P_{0} \rightarrow M \rightarrow 0$ where $P_{0}$ is generated in degree 0 and $P_{1}$ is generated in degree 1 . Obviously such a linear presentation is always minimal. A stronger notion is that of a linear module. We say that a graded module $M$ is linear if it has a minimal graded free resolution:

$$
\cdots P_{k} \rightarrow \cdots P_{1} \rightarrow P_{0} \rightarrow M \rightarrow 0
$$

where for each $i \geq 0$, the free module $P_{i}$ is generated in degree $i$. If we denote by $\mathcal{L}_{S}$ the subcategory of $\bmod ^{Z} S$ consisting of the modules with a linear presentation, we then have an exact equivalence of categories $\mathcal{L}_{S} \cong \mathcal{L}_{S / J^{2}}$ where $J$ denotes the maximal homogeneous ideal of $S$ ([6]). This equivalence is given by assigning to each module $M$ having a linear presentation over $S$ the module $M / J^{2} M$. We start with the following background result:

Lemma 1.1. An exact sequence $0 \rightarrow M \rightarrow E \rightarrow N \rightarrow 0$ in $\mathcal{L}_{S}$ splits if and only if the induced sequence $0 \rightarrow M / J^{2} M \rightarrow E / J^{2} E \rightarrow N / J^{2} N \rightarrow 0$ splits too.

Proof. To prove this, it is enough to show that a monomorphism $M \rightarrow E$ in $\mathcal{L}_{S}$ splits if and only if the induced map $M / J^{2} M \rightarrow E / J^{2} E$ is also a splittable monomorphism and one direction is trivial. For the other direction, recall that the category $\mathcal{L}_{S}$ is equivalent to the category $\mathcal{C}$ whose objects are graded morphisms $f: P_{1} \rightarrow P_{0}$ between free $S$-modules, where $P_{0}$ is generated in degree zero, $P_{1}$ is generated in degree one, and the degree one component of $f$ is a monomorphism from the degree one component of $P_{1}$ to the degree one component of $P_{0}$. A morphism in $\mathcal{C}$ from $f: P_{1} \rightarrow P_{0}$ to $g: Q_{1} \rightarrow Q_{0}$ is a pair $\left(u_{1}, u_{0}\right)$ of graded homomorphisms such that the diagram

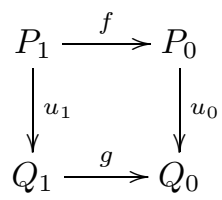

is commutative. Let $M$ and $E$ be two modules with linear presentations $P_{1} \stackrel{f}{\rightarrow} P_{0}$ and $Q_{1} \stackrel{g}{\rightarrow} Q_{0}$, and let $h: M \rightarrow E$ be a homomorphismm such that the induced map $\tilde{h}: M / J^{2} M \rightarrow E / J^{2} E$ is a splittable monomorphism. We have an exact 
commutative diagram:

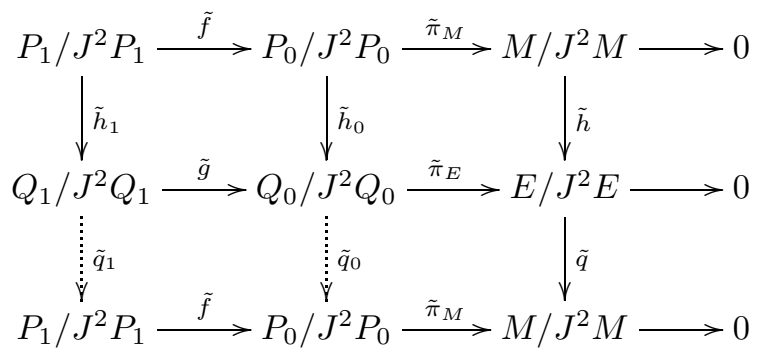

where $\tilde{q} \tilde{h}=1_{M / J^{2} M}$. Observe that the liftings $\tilde{q}_{1}$ and $\tilde{q}_{0}$ also have the property that $\tilde{q}_{1} \tilde{h}_{1}=1_{P_{1} / J^{2} P_{1}}$ and $\tilde{q}_{0} \tilde{h}_{0}=1_{P_{0} / J^{2} P_{0}}$. By lifting to $\mathcal{L}_{S}$ we obtain the following exact commutative diagram:

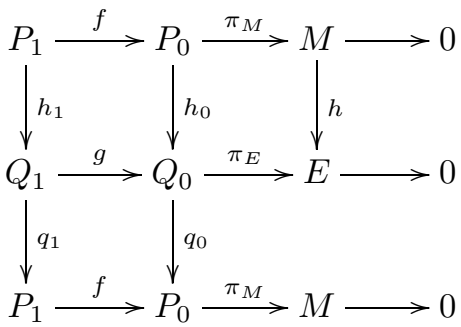

and we have that $q_{1} h_{1}=1_{P_{1}}$ and $q_{0} h_{0}=1_{P_{0}}$. There exists a unique homomorphism $l: E \rightarrow M$ such that $l \pi_{E}=\pi_{M} q_{0}$, and it follows immediately that $l h=1_{M}$.

We will also need the following results about graded modules over the polynomial algebra $S=\mathbb{C}\left[x_{0}, \ldots x_{n}\right]$ :

Lemma 1.2. Let $0 \rightarrow A \stackrel{f}{\rightarrow} B \stackrel{g}{\rightarrow} C \rightarrow 0$ be an exact sequence in $\bmod ^{\mathbb{Z}} S$ where $A$ is a linear module and $C$ is semisimple generated in a single degree $m \geq 0$. Then the sequence splits.

Proof. It suffices to show that the sequence $0 \rightarrow A_{\geq m} \stackrel{f_{\geq m}}{\longrightarrow} B \geq m \stackrel{g_{\geq m}}{\longrightarrow} C \rightarrow 0$ splits in the category of graded $S$-modules since we can easily construct a right inverse to $g$ from a right inverse to $g_{\geq m}$. Each of the modules $A_{\geq m}, B \geq m, C$ is an $m$-shift of a linear $S$-module, so by Koszul duality we obtain an exact sequence over the exterior algebra $R$ :

$$
0 \rightarrow \mathcal{E}(C) \rightarrow \mathcal{E}\left(B_{\geq m}\right) \rightarrow \mathcal{E}\left(A_{\geq m}\right) \rightarrow 0
$$

But $R$ is self-injective and $\mathcal{E}(C)$ is free, so this sequence splits. The lemma now follows immediately.

Lemma 1.3. Let $C$ be a linear $S$-module. Let $i$ be a positive integer and assume that $\operatorname{Ext}_{S}^{1}\left(C_{\geq i}, C\right)_{0}=0$. Then $\operatorname{Ext}_{S}^{1}\left(C_{\geq i}, C_{\geq i}\right)_{0}=0$. 
Proof. Assume to the contrary that $\operatorname{Ext}_{S}^{1}\left(C_{\geq i}, C_{\geq i}\right)_{0} \neq 0$. We have the following pushout diagram of graded modules:

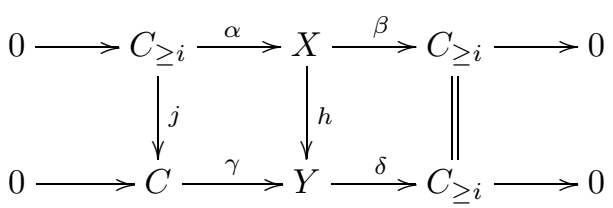

where $j$ is the inclusion map. The bottom sequence splits by assumption so we have homomorphisms $q: C_{\geq i} \rightarrow Y$ and $p: Y \rightarrow C$ such that $\delta q=1$ and $p \gamma=1$. But then each graded component of $\gamma, \delta$ splits so we obtain an induced split exact sequence $0 \rightarrow C_{\geq i} \rightarrow Y_{\geq i} \rightarrow C_{\geq i} \rightarrow 0$ and an induced commutative diagram:

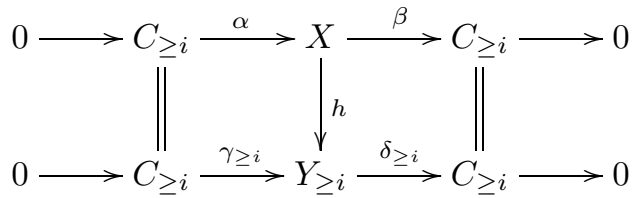

and since the two extensions represent the same element in $\mathrm{Ext}^{1}$ they both split.

The following result holds for an arbitrary Koszul algebra over the complex numbers.

Lemma 1.4. Let $\Gamma$ be a Koszul algebra and let $M \in \mathcal{L}_{\Gamma}$ be an indecomposable nonprojective $\Gamma$-module of Loewy length two having no graded self-extensions. Then $\operatorname{End}_{\Gamma}(M)_{0} \cong \mathbb{C}$.

Proof. The proof follows the same idea as the one given in [8], but we present it here for the reader's convenience. Assume that there exists a nonzero graded homomorphism $f: M \rightarrow M$ that is not an isomorphism, and let $N$ denote its image and $L$ its kernel. Furthermore, let $T$ denote the cokernel of the inclusion map $N \rightarrow M$. Note that $T$ is also generated in degree zero and that $L$ lives in degree 1 and possibly also in degree zero. Applying $\operatorname{Hom}_{\Gamma}(T,-)$ to the exact sequence $0 \rightarrow L \rightarrow M \rightarrow N \rightarrow 0$ and taking only the degree zero parts, we obtain an exact sequence

$$
\rightarrow \operatorname{Ext}_{\Gamma}^{1}(T, M)_{0} \rightarrow \operatorname{Ext}_{\Gamma}^{1}(T, N)_{0} \rightarrow \operatorname{Ext}_{\Gamma}^{2}(T, L)_{0} .
$$

But if

$$
\cdots \rightarrow P_{2} \rightarrow P_{1} \rightarrow P_{0} \rightarrow T \rightarrow 0
$$

is the beginning of a graded minimal projective resolution of $T$, the second term $P_{2}$ is generated in degrees 2 or higher; hence $\operatorname{Hom}_{\Gamma}\left(P_{2}, L\right)_{0}=0$ and therefore $\operatorname{Ext}_{\Gamma}^{2}(T, L)_{0}$ vanishes too. We obtain a surjection $\operatorname{Ext}_{\Gamma}^{1}(T, M)_{0} \rightarrow \operatorname{Ext}_{\Gamma}^{1}(T, N)_{0}$ and hence a commutative exact diagram of graded $\Gamma$-modules of Loewy length 2 and degree zero maps:

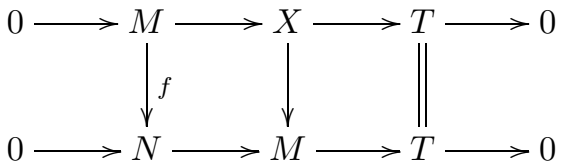

This pushout yields an exact sequence of graded $\Gamma$-modules:

$$
0 \rightarrow M \rightarrow N \oplus X \rightarrow M \rightarrow 0 .
$$


Our assumption on $M$ implies that this sequence must split. The Krull-RemakSchmidt theorem yields a contradiction, since $N \neq 0, M$ is indecomposable and $\ell(N)<\ell(M)$.

We can proceed now with the proof of our main result:

Theorem 1.5. Let $F$ be an indecomposable coherent sheaf over the projective space $\mathbf{P}^{n}$ such that $\operatorname{Ext}^{1}(F, F)=0$. Then, End $F \cong \mathbb{C}$.

Proof. By Serre's theorem, we may identify the category of coherent sheaves over $\mathbf{P}^{n}$ with the quotient category $Q \bmod ^{Z} S$ of $\bmod ^{Z} S$. Recall that the objects of $Q \bmod ^{Z} S$ are the objects of $\bmod ^{Z} S$ modulo the graded modules of finite dimension. Let $\widetilde{X}$ denote the sheafification of $X$, that is, the image of $X$ in the quotient category. If $X$ and $Y$ are two graded $S$-modules, then $\widetilde{X} \cong \widetilde{Y}$ in $Q \bmod ^{Z} S$ if and only if for some integer $k$, their truncations $X_{\geq k}$ and $Y_{\geq k}$ are isomorphic as graded $S$-modules. Now, let $F$ be an indecomposable sheaf with $\operatorname{Ext}^{1}(F, F)=0$. We may assume that $F=\widetilde{X}$ where $X$ is a linear $S$-module up to some shift ([1]) and that $X$ has no finite dimensional submodules. Then (see [10] for instance), $\operatorname{End}(F) \cong \lim _{\longrightarrow} \operatorname{Hom}_{S}\left(X_{\geq k}, X\right)_{0}$ and $\operatorname{Ext}^{1}(F, F) \cong \lim _{\longrightarrow} \operatorname{Ext}_{S}^{1}\left(X X_{\geq k}, X\right)_{0}$. For each $k$, we have an exact sequence of graded $S$-modules

$$
0 \rightarrow X_{\geq k+1} \rightarrow X_{\geq k} \rightarrow S_{k} \rightarrow 0
$$

where $S_{k}$ denotes the semisimple module $X_{\geq k} / X_{\geq k+1}$ concentrated in degree $k$. Applying $\operatorname{Hom}_{S}(-, X)$ and taking degrees, we obtain a long exact sequence

$$
\begin{gathered}
0 \rightarrow \operatorname{Hom}_{S}\left(X_{\geq k}, X\right)_{0} \rightarrow \operatorname{Hom}_{S}\left(X_{\geq k+1}, X\right)_{0} \rightarrow \operatorname{Ext}_{S}^{1}\left(S_{k}, X\right)_{0} \\
\rightarrow \operatorname{Ext}_{S}^{1}\left(X_{\geq k}, X\right)_{0} \rightarrow \operatorname{Ext}_{S}^{1}\left(X_{\geq k+1}, X\right)_{0}
\end{gathered}
$$

since $\operatorname{Hom}_{S}\left(S_{k}, X\right)_{0}=0$ by our assumption on $X$. $\operatorname{Ext}_{S}^{1}\left(S_{k}, X\right)_{0}$ also vanishes by Lemma 1.2, since having a nontrivial extension $0 \rightarrow X \rightarrow Y \rightarrow S_{k} \rightarrow 0$ yields a nonsplit exact sequence of degree $k$ shifts of linear modules $0 \rightarrow X_{\geq k} \rightarrow Y_{\geq k} \rightarrow$ $S_{k} \rightarrow 0$. Since for each $k, \operatorname{Hom}_{S}\left(X_{\geq k}, X\right)_{0}=\operatorname{End}_{S}\left(X_{\geq k}\right)$, we see that we have $\operatorname{End}(F) \cong \operatorname{End}_{S}(X)_{0} \cong \operatorname{End}_{S}\left(X_{\geq k}\right)_{0}$ for each $k \geq 0$. At the same time we have a sequence of embeddings

$$
\operatorname{Ext}_{S}^{1}(X, X)_{0} \hookrightarrow \cdots \hookrightarrow \operatorname{Ext}_{S}^{1}\left(X_{\geq k}, X\right)_{0} \hookrightarrow \operatorname{Ext}_{S}^{1}\left(X_{\geq k+1}, X\right)_{0} \hookrightarrow \cdots .
$$

Thus, the assumption that $\operatorname{Ext}^{1}(F, F)=0$ implies that for each $k$ we must have $\operatorname{Ext}_{S}^{1}\left(X_{\geq k}, X\right)_{0}=0$, in particular, $\operatorname{Ext}_{S}^{1}(X, X)_{0}=0 . X$ is a shift of a linear module so we may apply Lemma 1.1. We now apply Lemma 1.4 to conclude that the sheaf $F$ has trivial endomorphism ring.

\section{Auslander-Reiten components Containing Sheaves}

We start with the following general observation:

Lemma 2.1. Let $A \stackrel{\left[\begin{array}{l}u_{1} \\ u_{2}\end{array}\right]}{\longrightarrow} B_{1} \oplus B_{2} \stackrel{\left[\begin{array}{ll}v_{1} & v_{2}\end{array}\right]}{\longrightarrow} C \rightarrow A[1]$ be a triangle in a Krull-Schmidt triangulated category $\mathcal{T}$, and assume that $C$ is indecomposable and that both maps $v_{1}, v_{2}$ are nonzero. Then $\operatorname{Hom}_{\mathcal{T}}(A, C) \neq 0$. 
Proof. We prove that $v_{1} u_{1} \neq 0$. Assume that $v_{1} u_{1}=0$. Then, since the composition $\left[\begin{array}{ll}v_{1} & 0\end{array}\right]\left[\begin{array}{l}u_{1} \\ u_{2}\end{array}\right]: A \rightarrow C$ is equal to 0 , we have an induced homomorphism $\psi: C \rightarrow C$ such that $\psi\left[v_{1} v_{2}\right]=\left[v_{1} 0\right]$. Thus $\psi v_{1}=v_{1}$ and $\psi v_{2}=0$. Since $C$ is indecomposable, $\psi$ is either an isomorphism or it is nilpotent. It cannot be nilpotent since $v_{1} \neq 0$, and it cannot be an isomorphism since $v_{2} \neq 0$; hence a contradiction.

The BGG correspondence $\Phi$ is an exact equivalence of triangulated categories; hence for every graded $R$-module $M$, we have that $\Phi(\Omega M)=\Phi(M)[-1]$. An easy computation also shows that for each finitely generated graded module $M$ and integer $i$, we have that $\Phi(M(i)) \otimes \mathcal{O}(i)=\Phi(M)[i]$. It turns out that the modules corresponding to sheaves are located on the boundary of the Auslander-Reiten component containing them. This and more follows immediately from the next result by playing with the BGG correspondence.

Proposition 2.2. Let $B$ and $C$ be two $R$-modules corresponding to sheaves under the BGG correspondence. Then, for each $i>0 \underline{\operatorname{Hom}}_{R}\left(\tau^{i} B, C\right)_{0}=0$.

Proof. Since taking syzygies and graded shifts are self-equivalences of $\underline{\bmod }^{Z} R$, we have by 11 that $C$ is a weakly Koszul module, and so we can use the formula $\tau B=\Omega^{2} B(n+1)$, and so $\tau^{i} B=\Omega^{2 i} B(n i+i)$ for all $i \geq 1$. We then have

$\Phi\left(\tau^{i} B\right)=\Phi\left(\Omega^{2 i} B(n i+i)\right)=\Phi\left(\Omega^{2 i} B\right)[n i+i] \otimes \mathcal{O}(-n i-i)=\Phi(B)[n i-i] \otimes \mathcal{O}(-n i-i)$.

Applying the BGG correspondence, we obtain

$$
\begin{aligned}
\underline{\operatorname{Hom}}_{R}\left(\tau^{i} B, C\right)_{0} & \cong \operatorname{Hom}_{\mathcal{D}^{b}\left(\operatorname{coh}\left(\mathbf{P}^{n}\right)\right)}\left(\Phi\left(\tau^{i} B\right), \Phi(C)\right) \\
& =\operatorname{Hom}_{\mathcal{D}^{b}\left(\operatorname{coh}\left(\mathbf{P}^{n}\right)\right)}(\Phi(B)[n i-i] \otimes \mathcal{O}(-n i-i), \Phi(C)) \\
& \cong \operatorname{Hom}_{\mathcal{D}^{b}\left(\operatorname{coh}\left(\mathbf{P}^{n}\right)\right)}(\Phi(B)(-n i-i)[n i-i], \Phi(C))=0
\end{aligned}
$$

where the last equality holds since both $\Phi(C)$ and $\Phi(B)(-n i-i)$ are sheaves, $n \geq 2$, so $n i-i \geq 1$.

We have the following immediate consequence by letting $B=C$ and $i=1$ in the previous proposition and applying Lemma 2.1:

Corollary 2.3. Let $C$ be an indecomposable $R$-module corresponding to a sheaf under the $B G G$ correspondence, where $n \geq 2$. Then the Auslander-Reiten sequence ending at $C$ has indecomposable middle term.

We end with the following observation:

Proposition 2.4. Let $\mathcal{C}$ be a connected component of the Auslander-Reiten quiver of $\mathcal{D}^{b}\left(\operatorname{coh} \mathbf{P}^{n}\right)$ for $n>1$. Then $\mathcal{C}$ contains at most one indecomposable coherent sheaf.

Proof. By the previous results, if $\mathcal{C}$ contains a sheaf, then this sheaf must lie on the boundary. Under the BGG correspondence, two sheaves in $\mathcal{C}$ must correspond to two modules of the form $C$ and $\tau^{i} C$ for some $i \geq 1$. But by applying the BGG correspondence we get stalk complexes concentrated in different degrees; hence we obtain a contradiction. 


\section{REFERENCES}

[1] Luchezar L. Avramov and David Eisenbud, Regularity of modules over a Koszul algebra, J. Algebra 153 (1992), no. 1, 85-90, DOI 10.1016/0021-8693(92)90149-G. MR1195407|(93k:16018)

[2] Maurice Auslander, Idun Reiten, and Sverre O. Smalø, Representation theory of Artin algebras, Cambridge Studies in Advanced Mathematics, vol. 36, Cambridge University Press, Cambridge, 1995. MR1314422 (96c:16015)

[3] Bernstein, I.N., Gelfand, I.M., Gelfand, S.I., Algebraic bundles over $\mathbf{P}^{n}$ and problems in linear algebra, Funct. Anal. Appl., 12 (1979), 212-214.

[4] J.-M. Drezet, Fibrés exceptionnels et suite spectrale de Beilinson généralisée sur $\mathbf{P}_{2}(\mathbf{C})$, Math. Ann. 275 (1986), no. 1, 25-48, DOI 10.1007/BF01458581 (French). MR849052 (88b:14014)

[5] E. Graham Evans and Phillip Griffith, The syzygy problem, Ann. of Math. (2) 114 (1981), no. 2, 323-333, DOI 10.2307/1971296. MR632842(83i:13006)

[6] E. L. Green, R. Martinez-Villa, I. Reiten, Ø. Solberg, and D. Zacharia, On modules with linear presentations, J. Algebra 205 (1998), no. 2, 578-604, DOI 10.1006/jabr.1997.7402. MR 1632765 (99i:16075)

[7] Dieter Happel, Triangulated categories in the representation theory of finite-dimensional algebras, London Mathematical Society Lecture Note Series, vol. 119, Cambridge University Press, Cambridge, 1988. MR935124 (89e:16035)

[8] Dieter Happel and Claus Michael Ringel, Tilted algebras, Trans. Amer. Math. Soc. 274 (1982), no. 2, 399-443, DOI 10.2307/1999116. MR675063 (84d:16027)

[9] D. Huybrechts, Fourier-Mukai transforms in algebraic geometry, Oxford Mathematical Monographs, The Clarendon Press Oxford University Press, Oxford, 2006. MR2244106 (2007f:14013)

[10] R. Martínez-Villa, Koszul algebras and sheaves over projective space, arXiv:math.RT/ 0405538.

[11] Roberto Martínez-Villa and Dan Zacharia, Approximations with modules having linear resolutions, J. Algebra 266 (2003), no. 2, 671-697, DOI 10.1016/S0021-8693(03)00261-8. MR1995131 (2004h:16005)

[12] Roberto Martínez-Villa and Dan Zacharia, Auslander-Reiten sequences, locally free sheaves and Chebysheff polynomials, Compos. Math. 142 (2006), no. 2, 397-408, DOI 10.1112/S0010437X05001703. MR2218901(2006m:14022)

Fakultät für Mathematik, Technische Universität Chemnitz, 09107 Chemnitz, GerMANY

E-mail address: happel@mathematik.tu-chemnitz.de

Department of Mathematics, Syracuse University, Syracuse, New York 13244-0001

E-mail address: zacharia@syr.edu 